\title{
Systemic toxicity induced by aggregated layered double hydroxide nanoparticles
}

\author{
This article was published in the following Dove Press journal: \\ International Journal of Nanomedicine \\ 3 October 2017 \\ Number of times this article has been viewed
}

\section{Mina Yan \\ Chanzhen Yang \\ Binyao Huang \\ Zeqian Huang \\ Liangfeng Huang \\ Xuefei Zhang \\ Chunshun Zhao}

School of Pharmaceutical Sciences, Sun Yat-sen University, Guangzhou Higher Education Mega Center,

Guangzhou, China
Correspondence: Chunshun Zhao School of Pharmaceutical Sciences, Sun Yat-sen University, I 32 Waihuan East Road, Guangzhou Higher Education Mega Center, Guangzhou 510006, China Tel/fax +86 2039943 II8 Email zhaocs@mail.sysu.edu.cn

\begin{abstract}
Layered double hydroxide (LDH) nanoparticles are emerging as one of the promising nanomaterials for biomedical applications, but their systemic toxicity in vivo has received little attention. In the present study, the effects of inorganic nanoparticle aggregation on their systemic toxicity were examined. Remarkably, aggregation was observed after the mixing of naked LDH nanoparticles with saline or erythrocytes. Significant accumulation of the naked LDH nanoparticles in the lungs of mice was detected $1 \mathrm{~h}$ after intravenous administration, and the survival rate of mice was $0 \%$ after 6 repeated injections. Furthermore, flocculent precipitates in the alveoli and congestion in the lung interstitium were observed in the dead mice. However, lipid membrane-coated LDH nanoparticles would not form aggregates and could be injected intravenously $>6$ times without causing death. These findings suggested that repeated injections of LDH were lethal even at low dose ( $30 \mathrm{mg} / \mathrm{kg})$, and lipid membrane coating can be considered as an approach for reducing this risk.
\end{abstract}

Keywords: inorganic nanoparticles, aggregation, erythrocyte aggregation, repeated intravenous injection, pulmonary embolism, sudden death

\section{Introduction}

The era of nanotechnology began when the physicist and Nobel laureate Richard Feynman challenged the scientific community to think small in his 1959 lecture entitled "There's Plenty of Room at the Bottom."1 Nanomedicine is the application of nanotechnology aimed at improving the health of individuals through better diagnoses and treatments based on the significantly advantageous properties of nanoparticles. ${ }^{2,3}$

Before nanomedicine can be safely applied in a clinical setting, its biocompatibility, biodistribution, and clearance need to be carefully assessed. ${ }^{4-9}$ Nanoparticles produced using organic molecules as building blocks have been investigated in depth and are in various stages of preclinical and clinical development. ${ }^{10}$ Inorganic nanoparticles, with their material- and size-dependent physicochemical properties, which are not observed in traditional organic nanoparticles, have gained attention only recently.

Aggregation appears to be a ubiquitous phenomenon in all inorganic nanoparticles. ${ }^{11}$ The high concentration of ions and high protein content of biological media (such as blood, saliva, lung surfactant, or cell culture media) can both decrease the electrostatic repulsive forces between inorganic nanoparticles and cause the formation of aggregates. However, the influence of nanoparticle aggregation on the interactions of nanoparticles with molecules, cells, or tissues remains unclear. ${ }^{5,69,11}$

Layered double hydroxides (LDHs) are a class of anionic clays that possess a unique structure based on brucite-like layers, wherein part of the divalent cations are substituted for trivalent ones, thereby yielding positively charged sheets in a stacked position. ${ }^{12}$ 
Considerable scientific research has been conducted on the controlled synthesis and applications of LDH, and its progress in various fields of biomedicine has been summarized in a few reviews. ${ }^{5,13,14}$ Several researchers have conjugated a variety of drugs (including the anti-tuberculosis agent paraaminosalicylic acid; ${ }^{15}$ glucocorticoid drug betamethasone sodium phosphate; ${ }^{16}$ antihypertensive prodrug perindopril erbumine ${ }^{17}$ anticancer moieties such as chlorogenic acid, ${ }^{18}$ 10-hydroxycamptothecin, ${ }^{19}$ and protocatechuic acid; ${ }^{20}$ photosensitizer indole-3-acetic acid; ${ }^{21}$ and the poor water-soluble drug ibuprofen, ${ }^{22}$ as well as nucleic acids ${ }^{23}$ and enzymes ${ }^{24}$ ) into the interspaces of LDH nanoparticles and successfully delivered them into cells.

However, the toxicology of intravenously injected LDH nanoparticles has not yet been studied in depth; a majority of the extant studies were conducted only at the cell level. ${ }^{14}$ The low cytotoxicity in vitro offers no guarantee on the desired high biocompatibility in vivo. Furthermore, LDH nanoparticles were found to be intolerable when injected intravenously, and severe side effects (such as sudden death) were observed during the experimental period. ${ }^{25-28}$

LDH nanoparticles formed aggregates when exposed to physiological fluids, ${ }^{23,28}$ which might explain the side effects caused by their intravenous injection. Therefore, there is clearly a pressing need for further research aimed at exploring the relationship between the formation of aggregates and the systemic toxicology of intravenously injected LDH nanoparticles.

In the present study, the systemic toxicities of both naked LDH (nak-LDH) nanoparticles and lipid membrane-coated LDH (lip-LDH) nanoparticles were examined because of the demonstrated ability of the lipid membrane coating to prevent the formation of aggregates. ${ }^{28}$ Furthermore, the interaction between nanoparticles and electrolytes or erythrocytes was studied, the biodistribution and toxicology of intravenously injected nak-LDH or lip-LDH were evaluated, and the relationship between aggregate formation caused by $\mathrm{LDH}$ nanoparticles and their toxicology in vivo was discussed.

\section{Materials and methods \\ Materials}

Dioleoyl phosphate (sodium salt) (DOPA), distearoylphosphatidylethanolamine (DSPE), distearoylphosphocholine (DSPC), and cholesterol (CHOL) were purchased from Avanti Polar Lipids (Alabaster, AL, USA).

Sprague-Dawley (SD) rats $(220 \pm 20$ g) and Kunming (KM) mice ( $20 \pm 2 \mathrm{~g}$ ) were supplied by the Laboratory Animal Center of Sun Yat-sen University (Guangzhou, China). All the experimental procedures were approved and supervised according to the Guidance on the Treatment of Experimental Animals by the Institutional Animal Care and Use Committee of Sun Yat-sen University (Guangzhou, China).

\section{Preparation of LDH nanoparticles}

nak-LDH nanoparticles were prepared by a quick precipitation method and subsequent hydrothermal treatment. In brief, $3.0 \mathrm{mmol}$ of $\mathrm{MgCl}_{2}$ and $1.0 \mathrm{mmol}$ of $\mathrm{AlCl}_{3}$ were dissolved in $10 \mathrm{~mL}$ of deionized water. This salt solution was then rapidly (within $5 \mathrm{~s}$ ) added to a basic solution $(40 \mathrm{~mL})$ containing $6.0 \mathrm{mmol}$ of $\mathrm{NaOH}$ to generate the precipitate of nak-LDH. After the precipitate was stirred for $10 \mathrm{~min}$ in a $\mathrm{N}_{2}$ stream at $25^{\circ} \mathrm{C}$, it was collected via centrifugation and then washed twice. The washed precipitate was then manually dispersed in $20 \mathrm{~mL}$ of deionized water and placed in a 25 -mL Teflon-lined autoclave; this was followed by hydrothermal treatment at $100^{\circ} \mathrm{C}$ in an oven for $6 \mathrm{~h}$ to obtain the suspension of the nak-LDH nanoparticles.

lip-LDH nanoparticles were prepared by self-assembly. First, $3 \mathrm{mmol}$ of DOPA, $2.4 \mathrm{mmol}$ of DSPC, $2.0 \mathrm{mmol}$ of CHOL, and 0.6 mmol of DSPE-PEG ${ }_{2000}$ were dissolved in chloroform and dried under a $\mathrm{N}_{2}$ stream. Then, the lipid film was hydrated with $200 \mu \mathrm{L}$ deionized water to obtain an empty liposome suspension. The nak-LDH nanoparticle suspension $(200 \mu \mathrm{L})$ was added to the empty liposome suspension. The mixture was sonicated in a water bath by using a laboratory ultrasonic cleaning machine (SB-5200DTN; Ningbo Scientz Biotechnology Co., Ltd., Zhejiang, China) at $250 \mathrm{~W}$ for $10 \mathrm{~min}$.

\section{Preparation of isotonic nanoparticle suspension for in vivo study}

Both the nak-LDH and lip-LDH nanoparticle suspensions were prepared with deionized water that was hypotonic. An isotonic nak-LDH nanoparticle suspension was obtained by diluting the LDH nanoparticle suspension with an equal volume of $10 \%$ glucose solution. An isotonic lip-LDH nanoparticle suspension was obtained by hydrating the lipid materials with $200 \mu \mathrm{L}$ of a $10 \%$ glucose solution instead of deionized water before mixing with the nak-LDH nanoparticles.

\section{X-ray photoelectron spectroscopy (XPS)}

The relative amounts of magnesium and phosphorus in the nak-LDH and lip-LDH samples, the nak-LDH mixture, and the lipid materials were measured by using XPS. The mixture sample was prepared as follows: $200 \mu \mathrm{L}$ of the nak-LDH 
nanoparticle suspension was centrifuged, and the precipitate was washed twice with ethanol before being mixed with lipid materials (3.0 $\mu \mathrm{mol}$ of DOPA, $2.4 \mu \mathrm{mol}$ of DSPC, $2.0 \mu \mathrm{mol}$ of CHOL, and $0.6 \mu \mathrm{mol}$ of DSPE-PEG $\left.{ }_{2000}\right)$ in chloroform. The sample was dropped on a piece of cover glass. Chloroform was volatilized before the sample was transferred into the XPS instrument (ESCALab 250; Thermo Fisher Scientific, Inc., Waltham, MA, USA), where the analysis was performed.

\section{Solubility study}

The kinetics of the solubility of LDH was examined by evaluating the concentration of magnesium ions. For this purpose, $450 \mu \mathrm{L}$ of the nak-LDH (lip-LDH) nanoparticle suspension was placed in a dialysis bag. Then, the dialysis bag was soaked in a buffer solution $(280 \mathrm{~mL})$ of $\mathrm{pH} 5.0$ ( $\mathrm{pH}$ 7.4). The buffer solution was incubated at $37^{\circ} \mathrm{C}$ for $24 \mathrm{~h}$. The magnesium content of the buffer solution was analyzed at a predetermined time by flame atomic absorption spectrometry (FAAS).

\section{Stability study}

The stability of LDH was tested by mixing it with electrolytes or erythrocytes. The nak-LDH and lip-LDH nanoparticle suspensions were diluted 2-, 5-, or 10-fold with saline. Erythrocytes from mice were harvested and washed by centrifugation. The nak-LDH and lip-LDH nanoparticle suspensions were separately mixed with erythrocytes and diluted with a $5 \%$ glucose solution. The dilutions were dropped on glass substrates and observed using an inverted microscope. The size distribution of the dilutions was determined using a particle size analyzer (Mastersizer 2000; Malvern Instruments Ltd., Malvern, UK).

\section{Quantitative analysis of magnesium content of tissues}

KM mice were treated with 5\% glucose (control group), nakLDH, or lip-LDH (30 mg/kg LDH). The tested mice were sacrificed at 1 h, 24 h, and 7 days, and their organs (heart, liver, spleen, lungs, and kidneys) were excised. The collected organs (50-150 mg) were digested overnight with $2 \mathrm{~mL}$ of $70 \%$ nitric acid before being heated at $90^{\circ} \mathrm{C}$ for $3 \mathrm{~h} ; 0.2 \mathrm{~mL}$ of perchloric acid was then added, and the organs were heated for another $0.5 \mathrm{~h}$. The digested solution was diluted to $10 \mathrm{~mL}$ with deionized water for magnesium content quantification using FAAS25 (SOLAAR S4; Thermo Fisher Scientific). The measured magnesium contents of the nak-LDH and
lip-LDH groups were separately compared with that of the control group. The ratio $(\mathrm{R})$ of the measured content of each nanoparticle groups to that of the control group was calculated using the following formula:

$$
\mathrm{R}=\frac{\mathrm{C}_{\mathrm{NPs}}}{\mathrm{C}_{\mathrm{con}}}
$$

where $\mathrm{C}_{\mathrm{NPs}}$ denotes the measured magnesium content of the nak-LDH or lip-LDH group, and $\mathrm{C}_{\text {con }}$ denotes the measured magnesium content of the control group.

\section{Blood biochemical analysis}

SD rats were randomly divided into 3 groups, and each group was injected in the tail vein with the $5 \%$ glucose solution, nak-LDH, or lip-LDH (14 mg/kg LDH). Blood samples were collected at $24 \mathrm{~h}$ and then centrifuged to obtain plasma, which was stored at $-20^{\circ} \mathrm{C}$ until measurement. The blood biochemical markers were analyzed using a biochemical autoanalyzer (CX5; Beckman, Fullerton, CA, USA). The levels of alanine aminotransferase (ALT), aspartate aminotransferase (AST), and albumin (ALB) were examined to understand the liver function. The quantity of creatinine (CRE) was assessed to identify nephrotoxicity.

\section{Histopathological examination}

Histologic analysis for toxicity evaluation was performed by examining the morphological changes in the organs (heart, liver, spleen, lungs, and kidneys) of the mice. The samples were injected intravenously by tail vein injections to the KM mice at $30 \mathrm{mg} \mathrm{LDH} / \mathrm{kg}$. The organs (heart, liver, spleen, lungs, and kidneys) were collected $24 \mathrm{~h}$ after the first injection or immediately after the mice died. The obtained organ samples were fixed in $10 \%$ neutral buffered formalin for $24 \mathrm{~h}$. The formalin-fixed tissues were trimmed, embedded in paraffin, and sliced into 5-mm thick sections. These biopsy sections were stained with H\&E, and the morphology of the tissue on the slides was observed under an optical microscope (DM5000B; Leica, Wetzlar, Germany).

\section{Repeated injections}

To measure the acute toxicity, repeated injections were administered every other day. Six KM mice were randomly assigned to a group for the purpose of testing the nak-LDH or lip-LDH nanoparticles. A separate control group was also assigned. Samples were injected intravenously via tail vein injections to the KM mice at $30 \mathrm{mg} \mathrm{LDH} / \mathrm{kg}$. 


\section{Results}

\section{Characterization of nak-LDH and lip-LDH nanoparticles}

In our previous study, ${ }^{28,29}$ we synthesized nak-LDH and prepared lip-LDH for cancer therapy. The core-shell structure of the prepared lip-LDH was preliminarily confirmed, and the positive charge $(39.2 \pm 1.2 \mathrm{mV})$ of the synthesized nak-LDH was screened. The zeta potential of the lip-LDH was $-31.4 \pm 4.7 \mathrm{mV}$.

In the present study, in-depth information regarding nakLDH and lip-LDH was obtained. First, the surface elemental compositions of nak-LDH (Figure 1A), lip-LDH (Figure 1B), and a mixture of nak-LDH and lipid materials (Figure 1C) were examined. An obvious signal of magnesium and no signal of phosphorus were observed for the nak-LDH sample. In contrast, no obvious signal of magnesium was observed for the lip-LDH sample. This latter result could be explained using the detection principle of XPS. XPS detects only the surface elements of samples, and the sampling depth was 2-5 nm. For lip-LDH, nanoparticles were coated with lipid materials, and therefore, it was difficult to detect the presence of magnesium in this sample.

LDH nanoparticles can dissolve and release magnesium ions in water, especially in acidulous solution. However, the solubility of LDH nanoparticles might be altered by the lipid membrane coating. Therefore, the solubility of lip-LDH was investigated by evaluating the concentration of magnesium ions released from the lip-LDH nanoparticles in water solution. As shown in Figure 1D, when nak-LDH was soaked in PBS (pH 5.0), 97.67\% nak-LDH dissolved in $24 \mathrm{~h}$. Meanwhile, only $73.34 \%$ magnesium ions were detected after lip-LDH was soaked in PBS for the same time period; this indicates that the dissolution of LDH in the acidulous solution could be altered by the lipid membrane coating. Surprisingly, it was found that the lipid membrane coating could not hinder the release of magnesium ions in neutral solution. As shown in Figure 1D, the dissolution of LDH was almost the same in the nak-LDH and lip-LDH groups when the $\mathrm{pH}$ was 7.4. Both nak-LDH and lip-LDH released $40 \%$ magnesium ions in 1 day. These results suggest that the coated lipid membrane might have been discontinuous and perforated. The dissolution rate of LDH in neutral solution was extremely low, and magnesium ions gradually leaked out through the gaps between the lipid membranes at the same time; therefore, it was difficult to observe the blocking effect of the lipid membrane on the release of magnesium ions. However, the dissolution rate of LDH in the acidulous solution was much higher, and large amounts of magnesium ions could not leak out at a time.

\section{Stability study of LDH nanoparticles in biological medium}

The effect of electrolytes on the stability of nak-LDH and lip-LDH was tested in order to predict their safety in vivo. As shown in Figure 1E and F, the nak-LDH nanoparticles accumulated and precipitated after being diluted with saline, whereas the lip-LDH nanoparticles showed no obvious change after mixing. These results indicate that the lipid membrane coating might greatly improve the stability and safety of the LDH nanoparticles in vivo. The human body contains numerous electrolytes; according to the electric double layer theory, these electrolytes can destroy the stability of charged nanoparticles. This can explain why in the present study, positively charged nak-LDH nanoparticles accumulated and precipitated after being diluted with saline. This is dangerous because the occurrence of precipitation in the blood vessels when nanoparticles are injected intravenously would be life threatening. Fortunately, the lipid membrane coating was able to eliminate the influence of electrolytes on the LDH nanoparticles by screening the positive charge and forming a hydration layer to protect the nanoparticles. Therefore, the safety of LDH in vivo was improved by using the lipid membrane coating.

Erythrocytes occupied nearly half the volume of the blood, and the surface of their membranes contained negatively charged sialic acid, which could interact with positively charged LDH nanoparticles. The LDH nanoparticles were absorbed on the membranes of the erythrocytes, and they induced the formation of erythrocyte aggregates. As can be seen in Figure 2, slight erythrocyte aggregation was observed when erythrocytes and nak-LDH were mixed in the proportion range of 10:5-10:32 $(\mu \mathrm{L} / \mu \mathrm{L})$. Furthermore, severe erythrocyte aggregation was detected when the erythrocytes and nak-LDH were mixed in the proportion range of 10:40-10:50 $(\mu \mathrm{L} / \mu \mathrm{L})$. However, when the erythrocytes were mixed with lip-LDH, formation of any aggregate was difficult even when the mixing proportion was 10:50 $(\mu \mathrm{L} / \mu \mathrm{L})$ (Figure 3).

Severe erythrocyte aggregation occurred after the intravenous injection of nak-LDH. Moreover, the aggregates broke up by the shear force of the blood flow. The dynamic equilibrium between the formation and elimination of erythrocyte aggregates in the blood was essential to the toxicology effect of intravenously injected LDH nanoparticles. As shown in Figure 4A and B, erythrocytes were no larger than $14 \mu \mathrm{m}$ in size. However, particles with a size larger than $14 \mu \mathrm{m}$ were detected after nak-LDH was added to the suspension of the erythrocytes; this indicated the formation of aggregates 

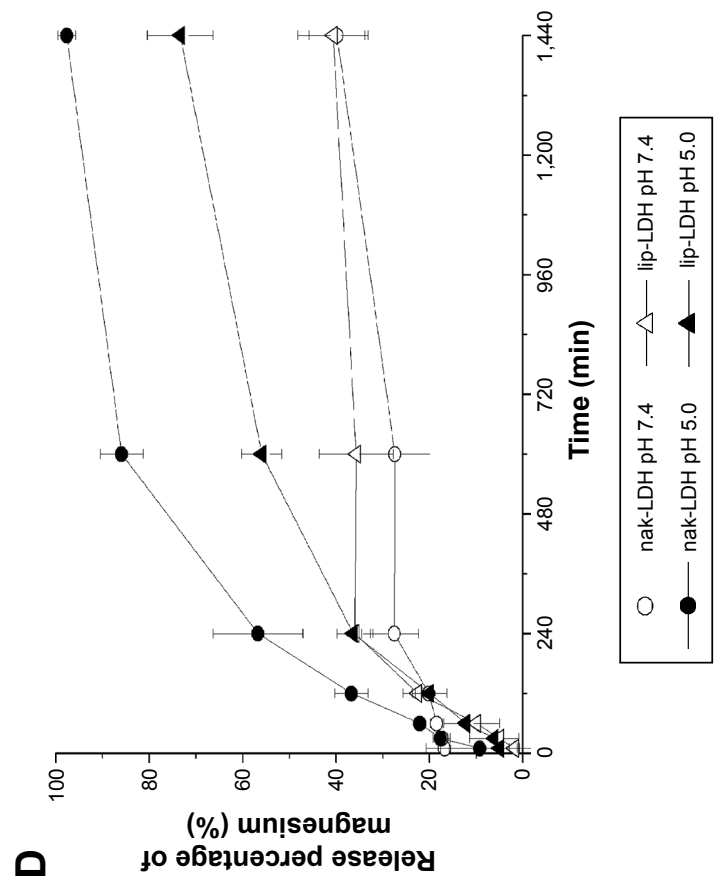

u
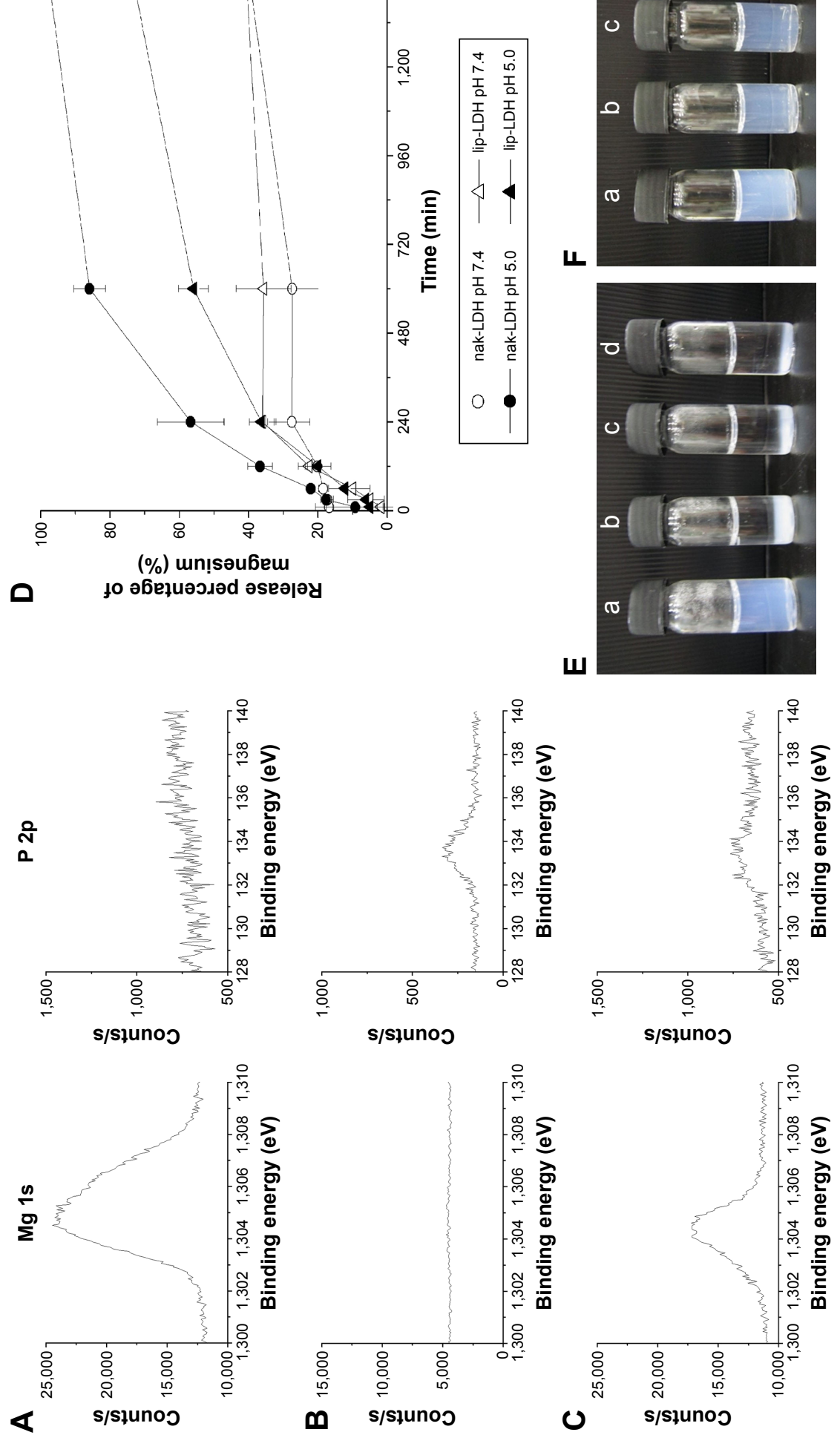

过

을 믕

두웡

음듬 문

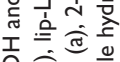

安苍递

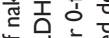

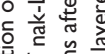

总

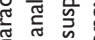

U⿺尢丶

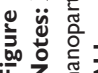




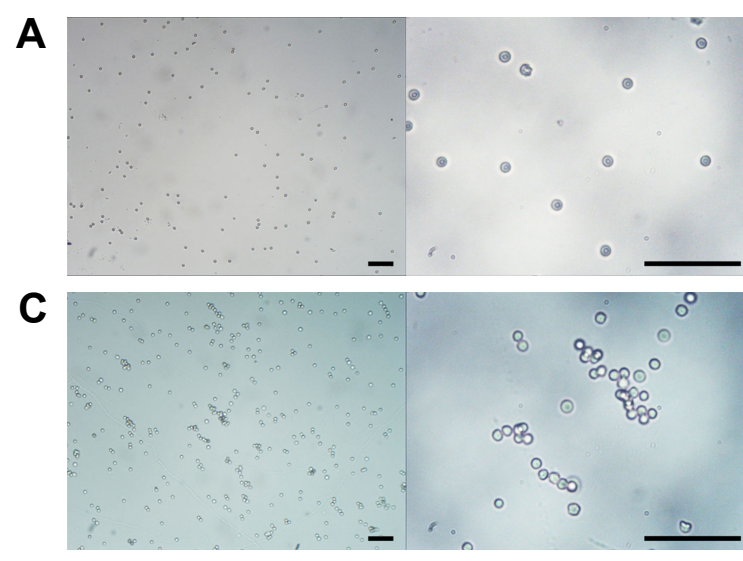

E

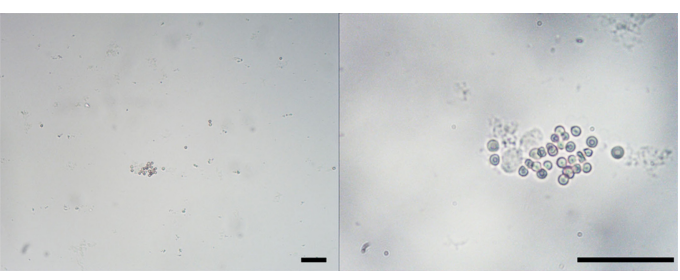

G

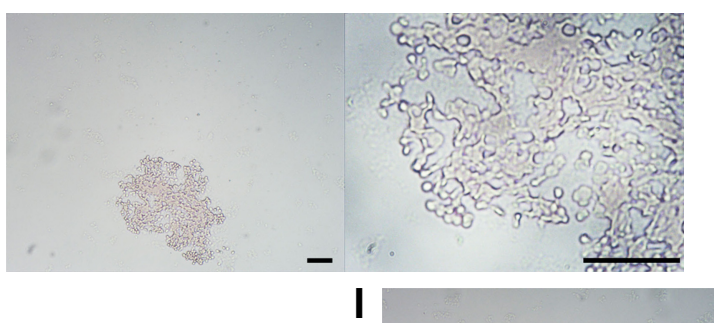

B

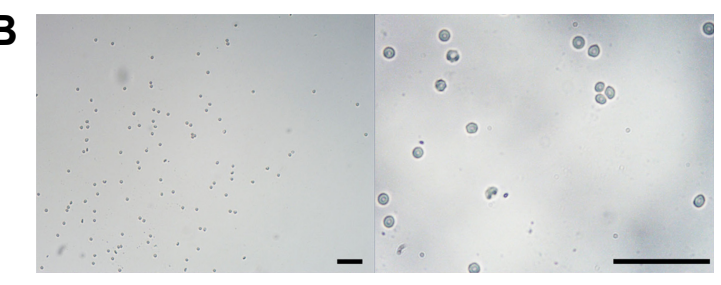

D

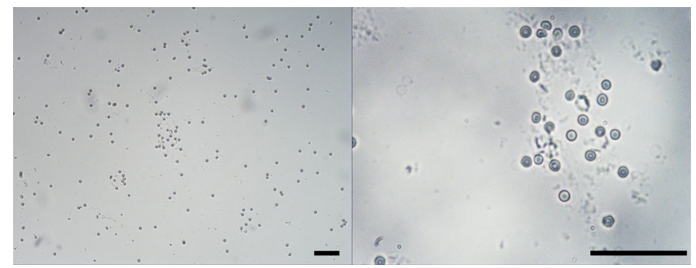

$\mathbf{F}$

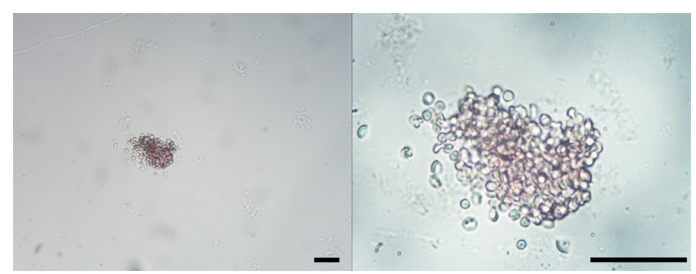

H

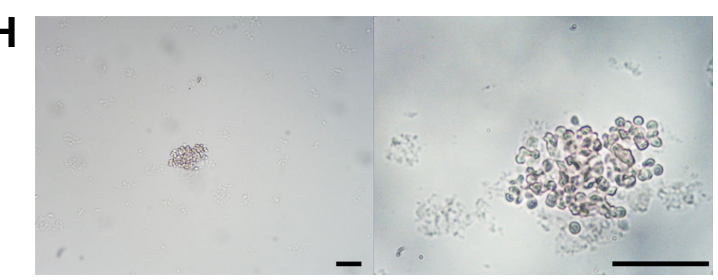

Figure 2 Optical microscope images of erythrocytes and nak-LDH mixed in the proportion of 10:0 (A), 10:2 (B), 10:5 (C), 10:25 (D), 10:32 (E), 10:40 (F), 10:50 (G), 10:60 $(\mathbf{H})$, and $10: 75(\mathbf{I})(\mu \mathrm{L} / \mu \mathrm{L})$.

Note: The scale bar is both $40 \mu \mathrm{m}$ for the two sets of images.

Abbreviations: LDH, layered double hydroxide; nak-LDH, naked LDH.

A

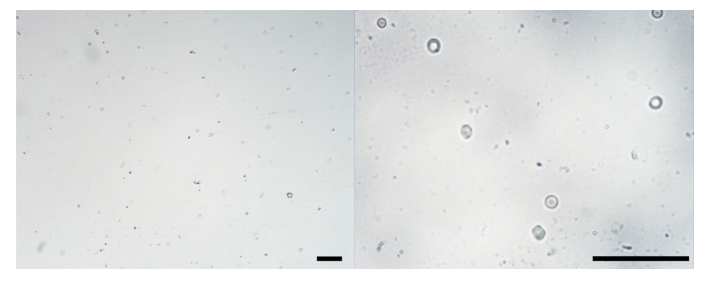

C

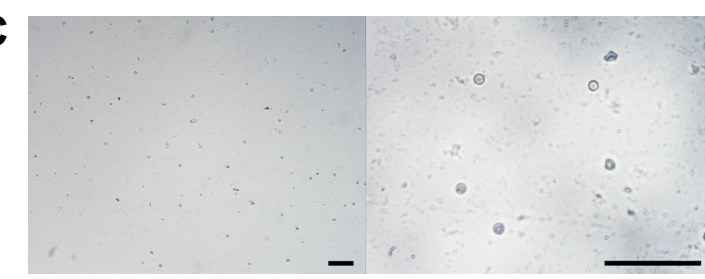

Figure 3 Optical microscope images of erythrocytes and lip-LDH mixed in the proportion of 10:25 (A), 10:50 (B), and I0:75 (C) $(\mu \mathrm{L} / \mu \mathrm{L})$.

Note: The scale bar is both $40 \mu \mathrm{m}$ for the two sets of images.

Abbreviations: LDH, layered double hydroxide; lip-LDH, lipid membrane-coated LDH. 
A

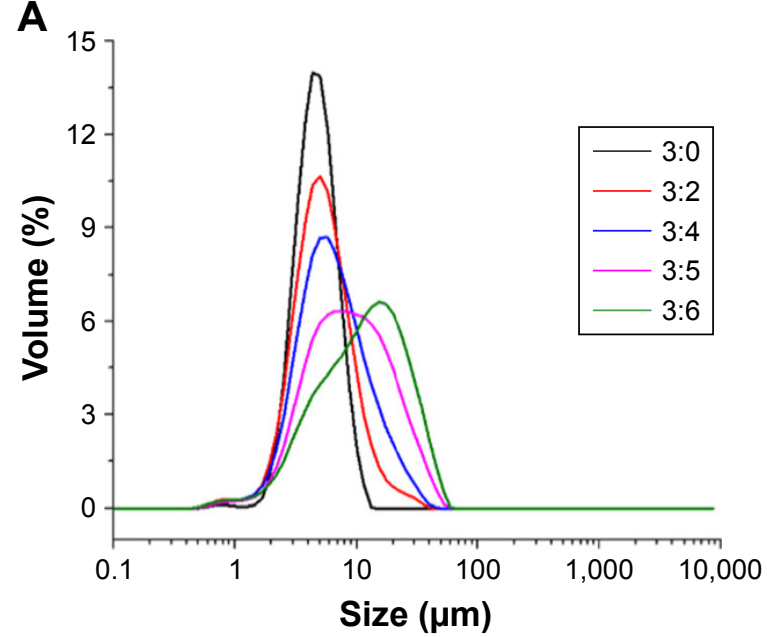

C

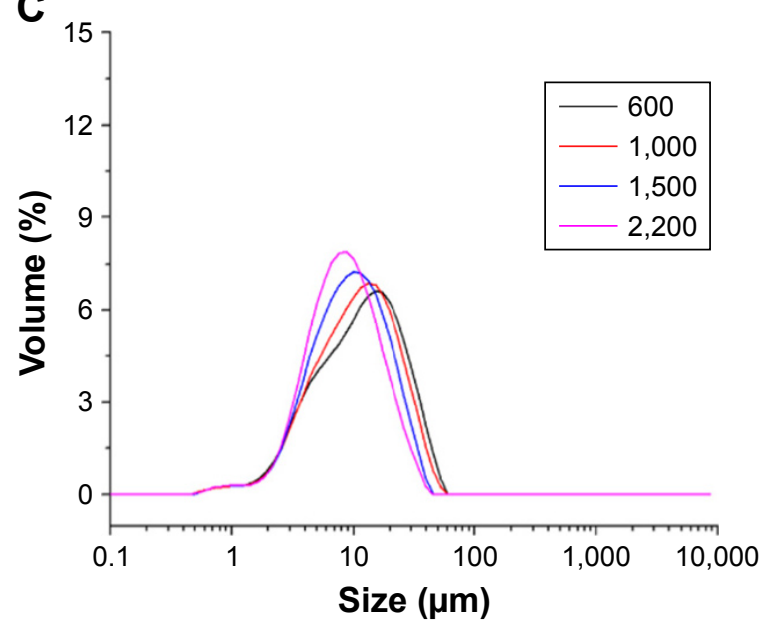

B
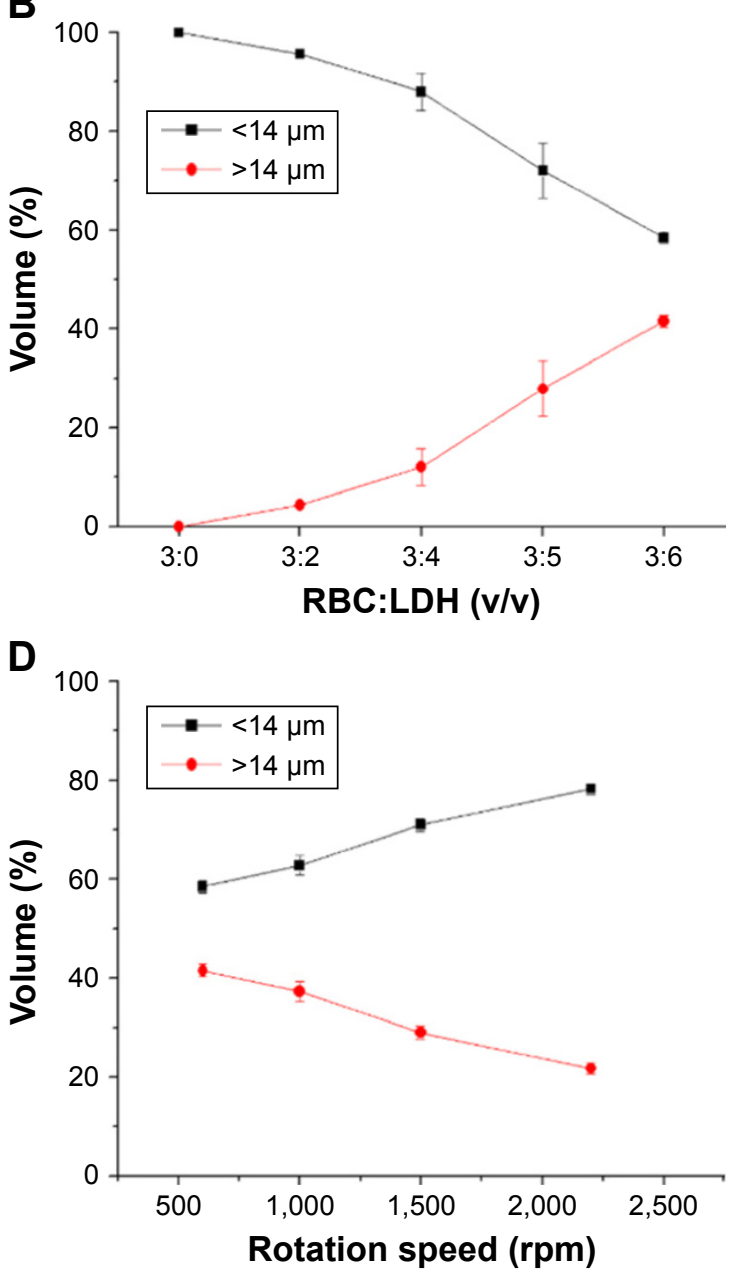

Figure 4 Size distribution (A) and peak area statistics (B) of erythrocytes mixed with nak-LDH in the proportion range of 3:0-3:6 ( $\mu \mathrm{L} / \mu \mathrm{L})$ under the rotational speed of $600 \mathrm{rpm}$. Size distribution (C) and peak area statistics (D) of erythrocytes mixed with nak-LDH in the proportion of 3:6 ( $\mu \mathrm{L} / \mu \mathrm{L})$ under the rotational speed ranged from 600 to $2,200 \mathrm{rpm}$.

Abbreviations: LDH, layered double hydroxide; nak-LDH, naked LDH; RBC, red blood cell.

(Figure 4A and B). Moreover, with an increase in the mixing speed, the proportion of aggregates with a size larger than $14 \mu \mathrm{m}$ decreased (Figure 4C and D), which indicated the elimination of the formed aggregates.

\section{Systemic toxicology study of LDH nanoparticles}

Magnesium ions play an important role in the physiological functions of living bodies, and LDH can release magnesium ions in physiological fluids. Therefore, in this study, FAAS was used to monitor changes in the magnesium content of tissues after the administration of nak-LDH nanoparticles or lip-LDH nanoparticles in mice. As shown in Figure 5A and B, after nak-LDH was administered, the magnesium content of the spleens and lungs increased significantly $1 \mathrm{~h}$ post injection $(p<0.05)$ and remained high for at least another $23 \mathrm{~h}(p<0.001)$. After lip-LDH group was administered, the magnesium content of the spleens increased $1 \mathrm{~h}$ post injection $(p<0.05)$, but was restored to the normal level in 1 day. Meanwhile, the magnesium content of the lungs was normal in the first hour after administration, and it increased significantly in $23 \mathrm{~h}(p<0.05)$. Furthermore, the magnesium content of the liver increased and remained high for $24 \mathrm{~h}$ $(p<0.05)$; however, this was not the case when nak-LDH group was administered.

The liver and kidneys are in charge of metabolism and excretion, respectively, in the body. In order to examine the potential effects of nanoparticles on these 2 vital organs after intravenous administration, the levels of ALT, AST, ALB, and CRE in the blood were detected and compared within the base values.

ALT and AST were present in the cytoplasm and nucleus, respectively, in the hepatocytes. Nominal damage can cause an increase in ALT in the blood, whereas severe damage can 


\section{A}
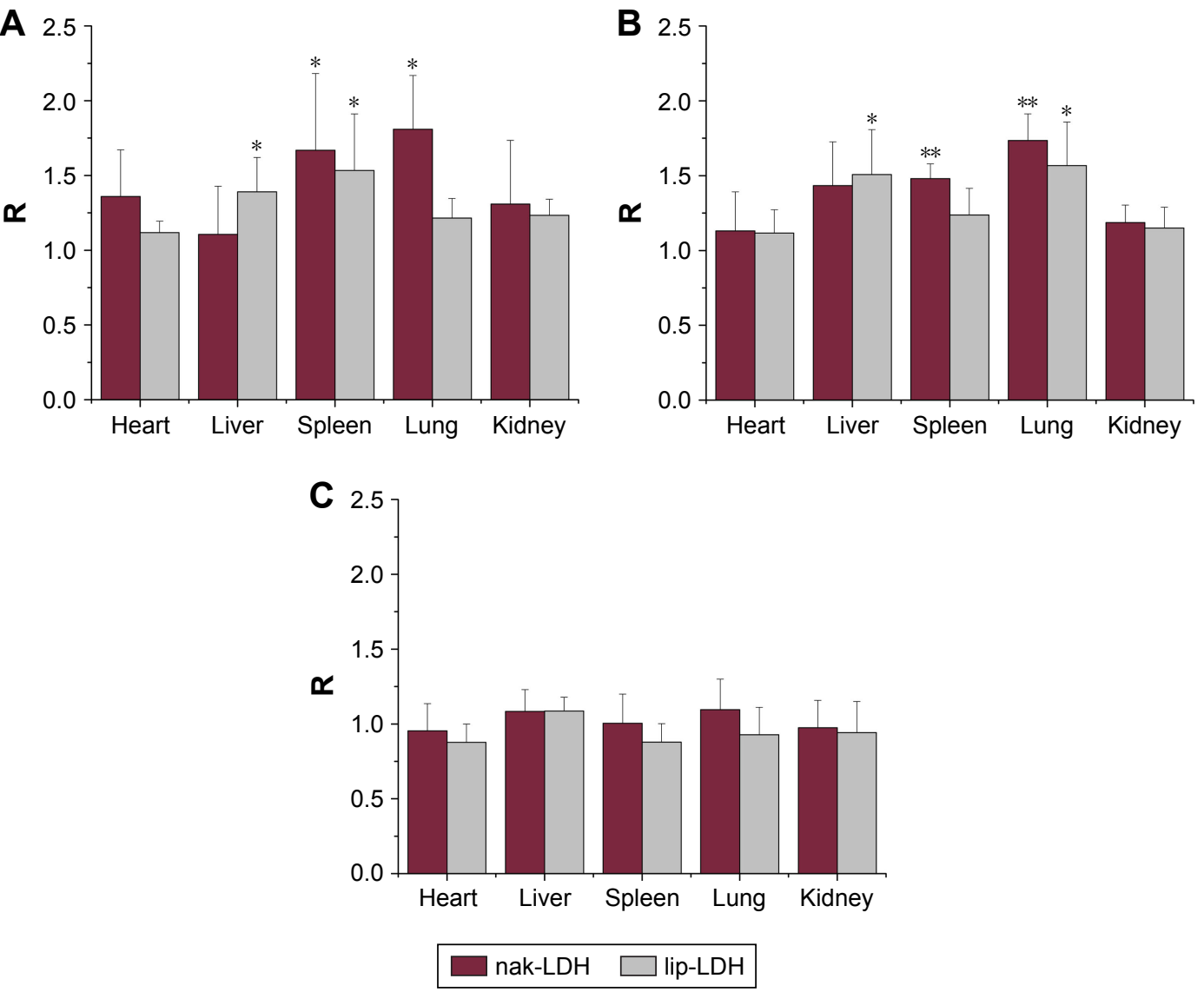

Figure $\mathbf{5}$ The ratio $(\mathrm{R})$ of the measured magnesium contents and base magnesium contents of heart, liver, spleens, lungs, and kidneys of KM mice I h (A), $24 \mathrm{~h}$ (B), and 7 days $(\mathbf{C})$ after administered with nak-LDH or lip-LDH $(n=6)$.

Note: $* p<0.05, * *_{p}<0.001$.

Abbreviations: LDH, layered double hydroxide; lip-LDH, lipid membrane-coated LDH; nak-LDH, naked LDH.

increase the level of AST. As a result, the AST/ALT ratio can reflect the degree of damage to the hepatocytes. As shown in Figure 6, the ALT and AST levels in the blood of the SD rats $24 \mathrm{~h}$ post injection of nak-LDH increased 1.24-fold and 1.5 -fold, respectively (Figure 6A and B). The AST/ALT ratio increased significantly $(p<0.001)$ (Figure 6C). In the case of injection of lip-LDH, even though the ALT and AST levels increased 1.3-fold and 1.25-fold, respectively (Figure 6A and B), there was no obvious difference in the AST/ALT ratio between the lip-LDH group and the control group (Figure 6C). After 6 days, the AST/ALT ratio for both the nak-LDH group and the lip-LDH group was at the same level as that of the control group, although the ALT level was slightly higher than normal for both the nak-LDH group and the lip-LDH group (Figure 6B). These results suggest that the nak-LDH nanoparticles caused much more severe damage to the hepatocytes than did the lip-LDH nanoparticles. This can be attributed to the affinity between the negatively charged lipid and the scavenger receptors expressed on the plasma membrane of Kupffer cells and sinusoidal endothelial cells in the liver. LDH nanoparticles coated with a negatively charged lipid membrane may have a tendency to accumulate in the Kupffer cells or liver sinusoidal endothelial cells instead of in the hepatocytes when passing through the liver along with the blood. As a result, damage to the hepatocytes was prevented to some degree.

ALB and CRE levels were detected in order to evaluate the degree of damage to the kidneys. ALB is produced in the liver and excreted via the kidneys. There are several possible reasons for a decrease in the ALB level in the blood: one reason is the lack of substrate for the production of ALB in the liver; another reason is that the production of ALB is blocked owing to liver damage; yet another reason is that the excretion of ALB is enhanced in the case of some kidney lesions. As a result, ALB can be used as an indicator of liver and kidney lesions simultaneously. However, CRE can only be used as an indicator of the degree of damage to the kidney, as it is excreted through the glomerulus. As shown in Figure 6D, the ALB level in the blood of SD rats remained unaffected by nak-LDH or lip-LDH injection both 

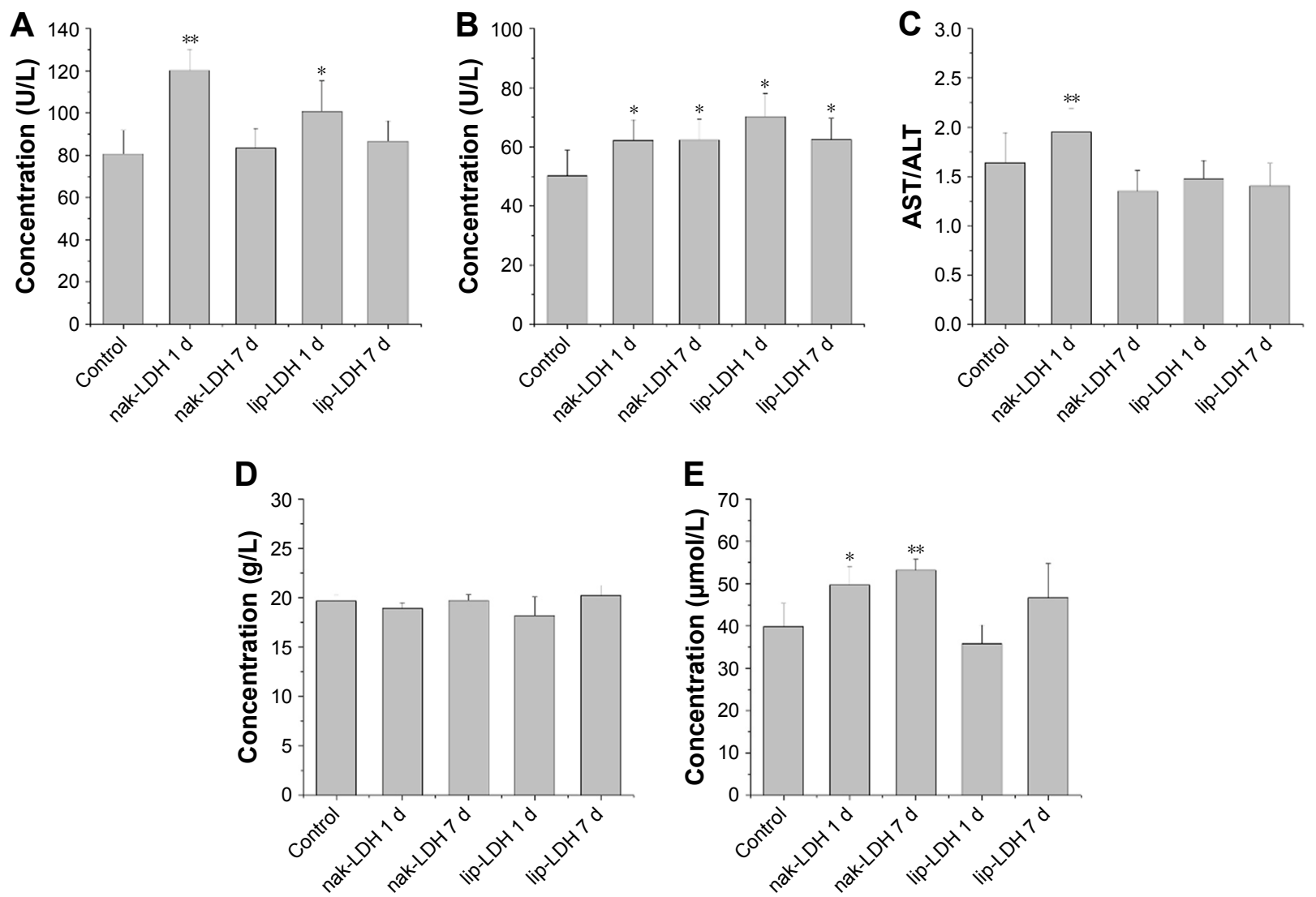

Figure 6 The level of ALT, AST, ALB and CRE in the blood was detected to evaluate the effects that the nanoparticles could have on the liver or kidneys after intravenous administration.

Notes: The level of AST (A), ALT (B), AST/ALT ratio (C), ALB (D), and CRE (E) of mice after administered with nak-LDH or lip-LDH nanoparticles ( $\mathrm{n}=6)$. ${ }^{*} p<0.05$, $* * p<0.001$.

Abbreviations: ALB, albumin; ALT, alanine aminotransferase; AST, aspartate aminotransferase; CRE, creatinine; LDH, layered double hydroxide; lip-LDH, lipid membranecoated LDH; nak-LDH, naked LDH.

$24 \mathrm{~h}$ and 7 days post injection. However, in the case of the nak-LDH group, the CRE level was increased significantly $(p<0.05) 24 \mathrm{~h}$ after it was administered, and it remained high even 6 days later $(p<0.001)$ (Figure 6E). These results suggest that serious damage to the glomerulus caused by the nak-LDH nanoparticles could be prevented through the use of nanoparticles coated with lipid membranes.

We further investigated via histopathological examination the toxic effects of nak-LDH and lip-LDH following their separate administration to mice. The investigation results indicated that neither nak-LDH nor lip-LDH had any obvious effect on the systemic toxicity after a single injection. No pathological changes in the heart, liver, spleen, lungs, or kidneys were observed $24 \mathrm{~h}$ post administration (Figure 7A-C).

\section{Repeated injection of LDH nanoparticles}

Single-dose toxicity of LDH was proven to be low, while repeated injections of drug-loaded LDH at the same dose were found to be lethal in our previous study. ${ }^{28}$ In this study, KM mice were intravenously injected with a nakLDH or lip-LDH nanoparticle suspension every other day. The survival rate was recorded to evaluate the safety of the use of nanoparticles. As shown in Figure 7D, the survival rate of mice injected with nak-LDH dropped to $0 \%$ after they were injected 6 times. In contrast, the mice injected with lip-LDH still exhibited a $100 \%$ survival rate after being injected 8 times. Moreover, flocculent precipitates in the alveoli and congestion in the lung interstitium were observed after repeated injection of the nak-LDH nanoparticles (Figure 7E).

\section{Discussion}

\section{Dynamic equilibrium of erythrocyte aggregation caused by LDH}

The dynamic equilibrium between the formation and elimination of erythrocyte aggregates was determined using the energies of bridging, electrostatic repulsion between the 


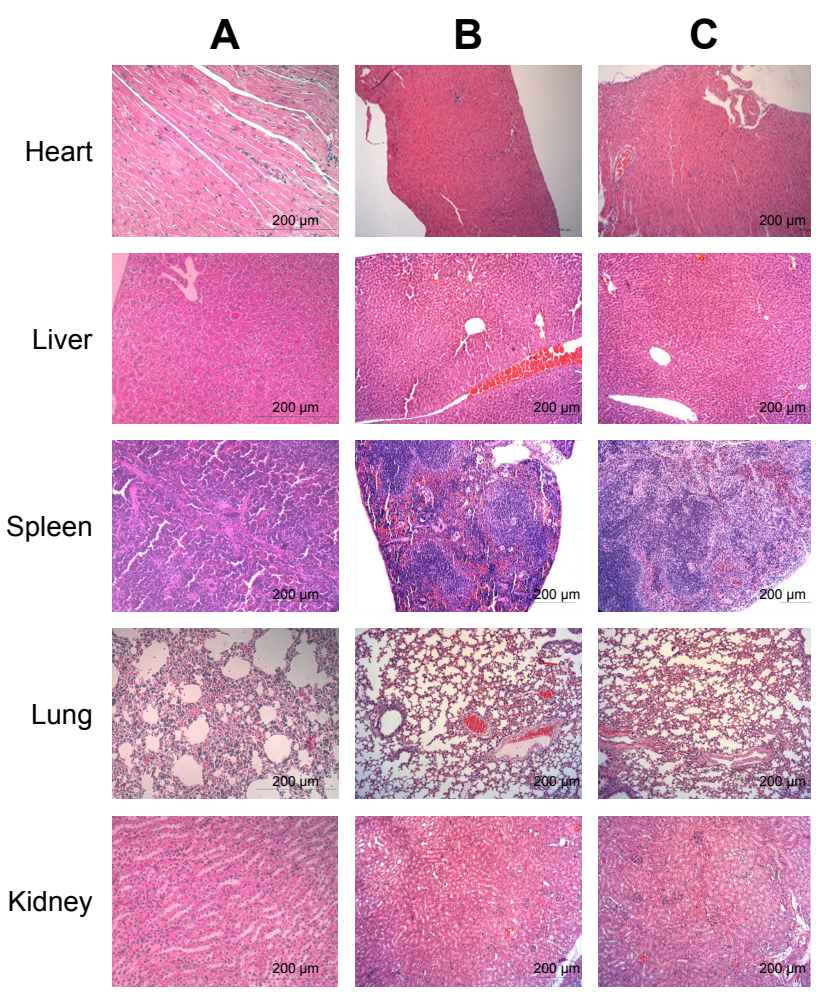

D
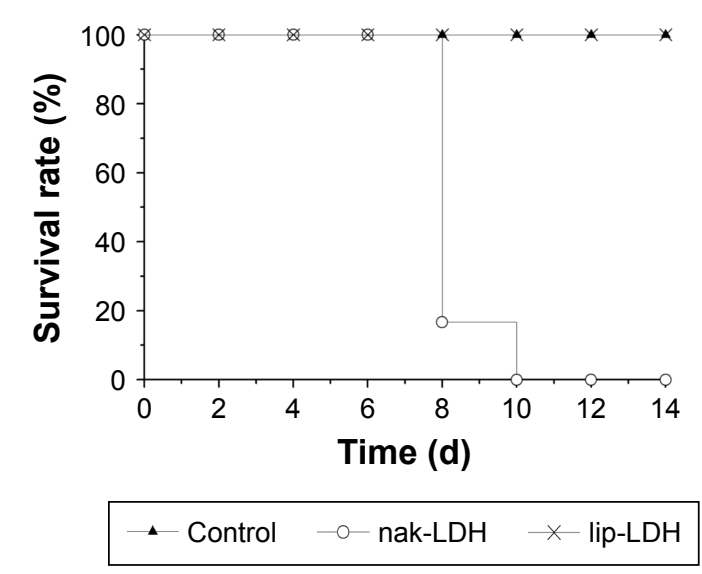

$\mathbf{E}$

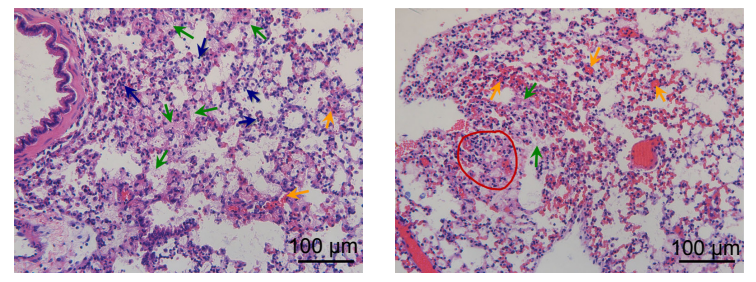

Figure 7 In vivo toxicology study of LDH nanoparticles.

Notes: Histopathology of the heart, liver, lungs, spleen, and kidneys in mice administered with 5\% glucose (A), nak-LDH (B), or lip-LDH (C). Survival rate of mice administered nak-LDH or lip-LDH every other day for 2 weeks $(n=6)(D)$. Histopathology of the lungs in mice administered with nak-LDH every other day till dead (E). Green arrow: flocculent precipitates in alveoli; blue arrow: infiltrating lymphocyte; yellow arrow: congestion in lung interstitium; red circle: thickened alveolar septum. Abbreviations: LDH, layered double hydroxide; lip-LDH, lipid membrane-coated LDH; nak-LDH, naked LDH.

erythrocytes, erythrocyte mobility, and shear force of the blood flow:

$$
E \mathrm{a}=E \mathrm{~b}-E \mathrm{c}-E \mathrm{~m}-E \mathrm{~s}
$$

where $E \mathrm{a}$ denotes the energy of the aggregate; $E \mathrm{~b}$ the energy of bridging; $E \mathrm{c}$ the energy of electrostatic repulsion; $E \mathrm{~m}$ the energy of mobility of erythrocytes; and Es the energy of the shear force of the blood flow.

When positively charged nak-LDH nanoparticles were added to the erythrocyte suspension, the repulsive force between the erythrocytes decreased (Figure 8A). As a result, the erythrocyte aggregates formed easily (Figure 4A and B). However, the shear force increased as the mixing speed increased, and the aggregated erythrocytes separated again (Figures 4C and D, and 8B).

\section{Biodistribution of LDH affected by surface modification}

The biodistribution of the nanoparticles was greatly affected by the mononuclear phagocytic system in the bodies. Nanoparticles were internalized by the phagocytes in the spleens, which led to accumulation of the nanoparticles. Moreover, negatively charged liposomes specifically bound to the scavenger receptors expressed on the plasma membrane of the Kupffer cells and sinusoidal endothelial cells in the liver. This could explain the increase in the magnesium content of the liver when the LDH nanoparticles were coated with a negatively charged lipid membrane (Figure 5A and B).

The stability of the nanoparticles in the blood was another factor that could have impacted their biodistribution. The results of the aforedescribed stability study (Figures 1E and 2) suggest that nak-LDH had a tendency to precipitate when mixed with electrolytes and erythrocytes. Numerous electrolytes and erythrocytes were present in the blood, which could have caused precipitation after the intravenous injection of nak-LDH. The precipitate could have flowed with the blood and blocked the capillaries in the lungs. However, the stability of the LDH nanoparticles improved when they were coated with the lipid membrane, and they could accumulate only in the lungs after the lipid membrane was peeled off. This could explain the increase in the magnesium content of the lungs in the case of both the nak-LDH group (Figure 5A) and the lip-LDH group (Figure 5B). 
A

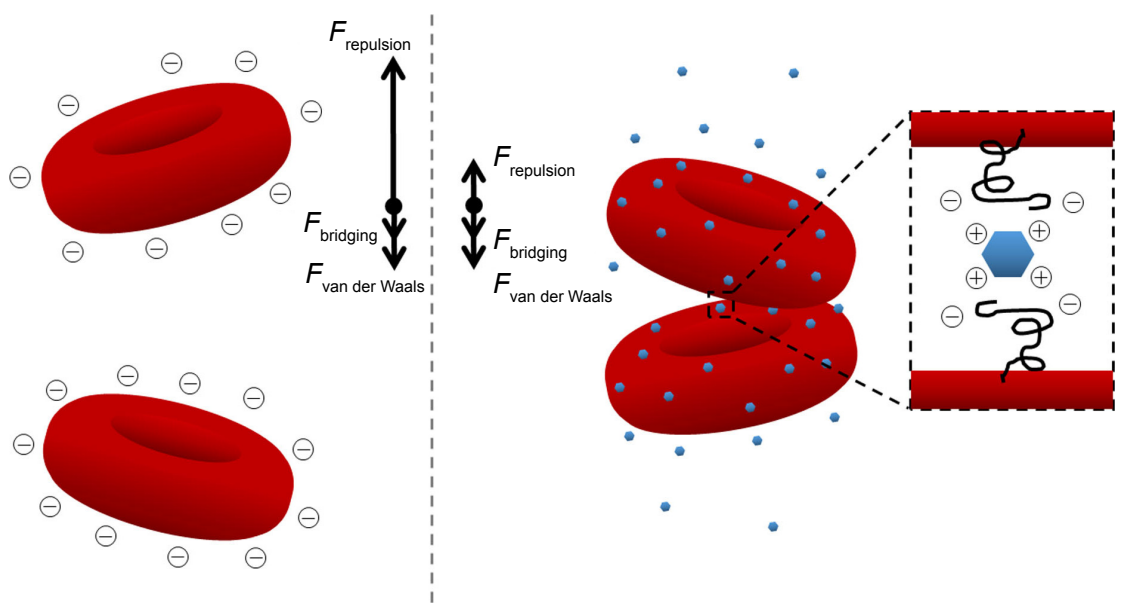

B
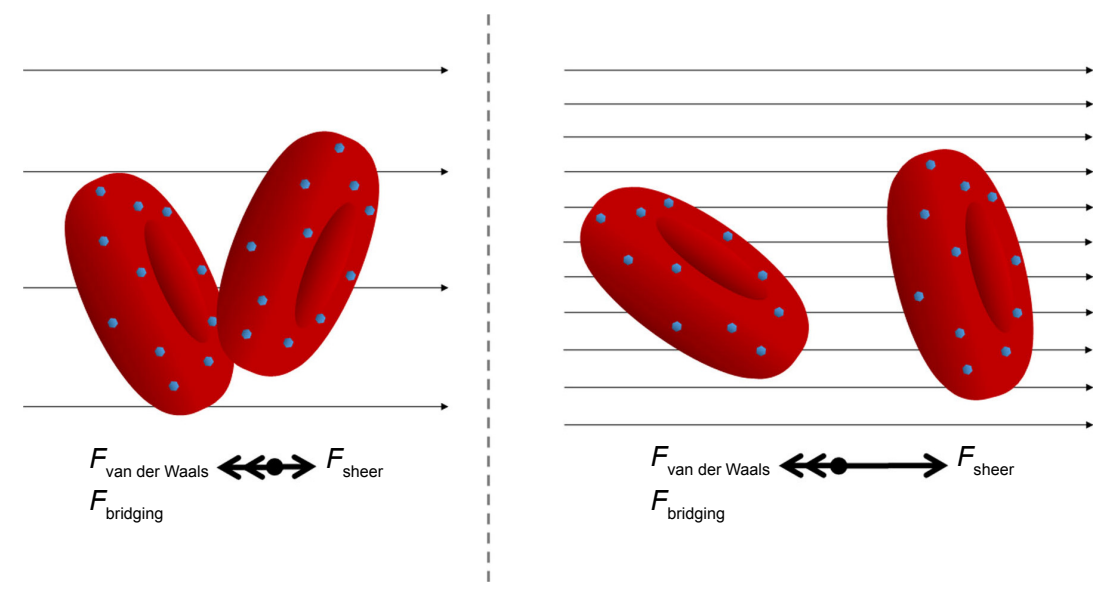

Figure 8 Formation of erythrocyte aggregation caused by positively charged nak-LDH nanoparticles (A). Elimination of erythrocyte aggregation caused by sheer force of blood flow (B).

Abbreviations: LDH, layered double hydroxide; nak-LDH, naked LDH.

\section{Systemic toxicity induced by repeated intravenous injection of LDH}

To the best of our knowledge, few in vivo studies reported the toxicity of LDH, especially the repeated-dose toxicity. As a result, the toxicity of $\mathrm{LDH}$ could be underestimated.

In this study, we show that the nak-LDH nanoparticles can cause death immediately after being intravenously injected in $\mathrm{KM}$ mice even at low dose $(30 \mathrm{mg} / \mathrm{kg})$, and this phenomenon can be eliminated if the nak-LDH nanoparticles are coated with lipid membranes. We believe that the death caused by the LDH nanoparticles can be attributed to their instability; that is, they may form precipitates and block the capillaries in the lungs after administration.

LDH nanoparticles can form precipitates immediately after being injected into the blood vessels, owing to the large number of electrolytes and negatively charged proteins present in the blood. In our previous study, ${ }^{28}$ we confirmed that LDH precipitated immediately after being mixed with calf serum, which could be the result of the interaction between the LDH and the serum proteins. ${ }^{23}$ Furthermore, the data obtained in this study also support our viewpoint that precipitation occurred immediately after the LDH nanoparticle suspension was diluted with saline (Figure 1E) or after the LDH nanoparticles were mixed with erythrocytes (Figures 2 and 4). The formed precipitates blocked the capillaries in the lungs and dramatically raised the risk of death. The data in Figure $5 \mathrm{~A}$ show that the magnesium content of the lungs rose significantly $(p<0.05) 1 \mathrm{~h}$ after administration of nak-LDH, which indicates accumulation of LDH in the lungs.

However, it is difficult to block all the capillaries with a single injection of LDH, because of the massive number of capillaries in the lungs. Moreover, the precipitates dispersed again upon being diluted with the blood during the time in which it flowed through the vessels before it reached the lungs. This could explain why the magnesium content of the lungs was restored to the normal level $24 \mathrm{~h}$ post injection of nak-LDH (Figure 5B). As can be observed in Figure 7D, no mice died after the first injection of nak-LDH, but 5 mice 
died after 5 repeated injections. These results suggest that severe side effects of LDH might only occur after repeated injections. As a result, systemic toxicity of LDH would be underestimated.

Positively charged drug carriers are more likely to induce higher toxicity than neutral or negatively charged drug carriers. It has been observed by other researchers that positively charged poly(amidoamine) (PAMAM) nanoparticles cause death after intravenous injection. ${ }^{30}$ The reason for immediate death after the injection of PAMAM was considered to be the coagulation caused by the nanoparticles, whose mechanism might be the interaction between the amides of PAMAM and the scissile amide bond in fibrinogen. In our study, however, coagulation was not observed, and no amide was present in the LDH nanoparticles. Therefore, we suggest that different reasons explain why inorganic nanoparticles and organic nanoparticles result in the death of mice, even though both types of nanoparticles carry a positive surface charge.

To the best of our knowledge, death caused by LDH nanoparticles has thus far not been reported. Precipitation might also occur in the case of other types of inorganic nanoparticles. There are at least 4 factors that may influence the formation of an embolism caused by inorganic nanoparticles in the lungs: the size of the nanoparticles, their concentration, the duration of the injections, and the diameter of the capillaries in the lungs.

\section{Conclusion}

In this study, the effects of inorganic nanoparticle aggregation on their systemic toxicity were examined. Remarkably, aggregation was observed after nak-LDH nanoparticles were mixed with saline or erythrocytes. Significant accumulation of the nak-LDH nanoparticles in the lungs was detected $1 \mathrm{~h}$ after their intravenous administration, and the survival rate of the mice was $0 \%$ after 6 repeated injections. Furthermore, flocculent precipitates in the alveoli and congestion in the lung interstitium were observed in the dead mice. These results suggest that a pulmonary embolism could be the reason for the sudden death, and this might only occur after repeated injections. As a result, systemic toxicity of LDH would be underestimated. Meanwhile, the lip-LDH nanoparticles accumulated in the liver instead of the lungs $1 \mathrm{~h}$ after injection, and no sudden death was observed post the repeated administration. Therefore, we conclude that aggregate formation of LDH nanoparticles in a biological medium greatly affects their biodistribution and increases their systemic toxicology in vivo, and the application of a lipid membrane coating can be used as an approach to change their biodistribution and decrease their systemic toxicology.

\section{Acknowledgments}

This research was supported by the Guangdong Medical Research Foundation, Guangzhou, China (grant number, A2016087), and partly by the National Natural Science Foundation of China (grant number 81673369/H3008). We thank Elsevier Webshop for the language editing service provided during the preparation of this manuscript.

\section{Disclosure}

The authors report no conflicts of interest in this work.

\section{References}

1. Maynard AD, Aitken RJ, Butz T, et al. Safe handling of nanotechnology. Nature. 2006;444:267-269.

2. Stirland DL, Nichols JW, Miura S, Bae YH. Mind the gap: a survey of how cancer drug carriers are susceptible to the gap between research and practice. J Control Release. 2013;172(3):1045-1064.

3. Sharifi S, Behzadi S, Laurent S, Forrest ML, Stroeve P, Mahmoudi M. Toxicity of nanomaterials. Chem Soc Rev. 2012;41(6):2323-2343.

4. Liu T, Li L, Teng X, et al. Single and repeated dose toxicity of mesoporous hollow silica nanoparticles in intravenously exposed mice. Biomaterials. 2011;32(6):1657-1668

5. Choi SJ, Lee JK, Jeong J, Choy JH. Toxicity evaluation of inorganic nanoparticles: considerations and challenges. Mol Cell Toxicol. 2013; 9(3):205-210.

6. Nel AE, Madler L, Velegol D, et al. Understanding biophysicochemical interactions at the nano-bio interface. Nat Mater. 2009;8(7):543-557.

7. Zhu M, Nie G, Meng H, Xia T, Nel A, Zhao Y. Physicochemical properties determine nanomaterial cellular uptake, transport, and fate. Acc Chem Res. 2013;46(3):622-631.

8. Wang B, He X, Zhang Z, Zhao Y, Feng W. Metabolism of nanomaterials in vivo: blood circulation and organ clearance. Acc Chem Res. 2013; 46(3):761-769.

9. Shi J, Kantoff PW, Wooster R, Farokhzad OC. Cancer nanomedicine: progress, challenges and opportunities. Nat Rev Cancer. 2017;17(1): $20-37$.

10. Huang HC, Barua S, Sharma G, Dey SK, Rege K. Inorganic nanoparticles for cancer imaging and therapy. J Control Release. 2011;155(3): 344-357.

11. Albanese A, Chan WC. Effect of gold nanoparticle aggregation on cell uptake and toxicity. ACS Nano. 2011;5(7):5478-5489.

12. Choi SJ, Choy JH. Layered double hydroxide nanoparticles as targetspecific delivery carriers: uptake mechanism and toxicity. Nanomedicine (Lond). 2011;6(5):803-814.

13. Kuthati Y, Kankala RK, Lee CH. Layered double hydroxide nanoparticles for biomedical applications: current status and recent prospects. Appl Clay Sci. 2015;112-113:100-116.

14. Kura AU, Hussein MZ, Fakurazi S, Arulselvan P. Layered double hydroxide nanocomposite for drug delivery systems; bio-distribution, toxicity and drug activity enhancement. Chem Cent J. 2014;8(1):47.

15. Saifullah B, Hussein MZ, Hussein-Al-Ali SH, Arulselvan P, Fakurazi S. Antituberculosis nanodelivery system with controlled-release properties based on para-amino salicylate-zinc aluminum-layered doublehydroxide nanocomposites. Drug Des Devel Ther. 2013;7:1365-1375.

16. Zhang Y, Wu X, Li H, Du N, Song S, Hou W. Preparation and characterization of (betamethasone sodium phosphate intercalated layered double hydroxide)@liposome nanocomposites. Colloids Surf A Physicochem Eng Asp. 2017;529:824-831.

17. Hussein Al Ali SH, Al-Qubaisi M, Hussein MZ, Ismail M, Zainal Z, Hakim MN. Comparative study of Mg/Al- and Zn/Al-layered double hydroxide-perindopril erbumine nanocomposites for inhibition of angiotensin-converting enzyme. Int J Nanomedicine. 2012;7: $4251-4262$. 
18. Barahuie F, Hussein MZ, Arulselvan P, Fakurazi S, Zainal Z. Drug delivery system for an anticancer agent, chlorogenate-Zn/Al-layered double hydroxide nanohybrid synthesised using direct co-precipitation and ion exchange methods. J Solid State Chem. 2014;217:31-41.

19. Zhang Y, Wu X, Mi Y, Li H, Hou W. Engineering of (10-hydroxycamptothecin intercalated layered double hydroxide)@liposome nanocomposites with excellent water dispersity. J Phys Chem Solids. 2017;108: 125-132.

20. Barahuie F, Hussein MZ, Gani SA, Fakurazi S, Zainal Z. Synthesis of protocatechuic acid-zinc/aluminium-layered double hydroxide nanocomposite as an anticancer nanodelivery system. J Solid State Chem. 2015;221:21-31

21. Kankala RK, Kuthati Y, Liu CL, Lee CH. Hierarchical coated metal hydroxide nanoconstructs as potential controlled release carriers of photosensitizer for skin melanoma. RSC Adv. 2015;5:42666-42680.

22. DeLeon VH, Nguyen TD, Nar M, D’Souza NA, Golden TD. Polymer nanocomposites for improved drug delivery efficiency. Mater Chem Phys. 2012;132(2-3):409-415.

23. Dong H, Parekh HS, Xu ZP. Enhanced cellular delivery and biocompatibility of a small layered double hydroxide-liposome composite system. Pharmaceutics. 2014;6(4):584-598.

24. Zou N, Plank J. Intercalation of papain enzyme into hydrotalcite type layered double hydroxide. J Phys Chem Solids. 2012;73(9): 1127-1130.
25. Kwak SY, Kriven WM, Wallig MA, Choy JH. Inorganic delivery vector for intravenous injection. Biomaterials. 2004;25(28):5995-6001.

26. Kuo YM, Kuthati Y, Kankala RK, et al. Layered double hydroxide nanoparticles to enhance organ-specific targeting and the anti-proliferative effect of cisplatin. J Mater Chem B. 2015;3(17):3447-3458.

27. Wei PR, Cheng SH, Liao WN, Kao KC, Weng CF, Lee CH. Synthesis of chitosan-coated near-infrared layered double hydroxide nanoparticles for in vivo optical imaging. J Mater Chem. 2012;22(12):5503-5513.

28. Yan M, Zhang Z, Cui S, et al. Improvement of pharmacokinetic and antitumor activity of layered double hydroxide nanoparticles by coating with PEGylated phospholipid membrane. Int J Nanomedicine. 2014; 9:4867-4878.

29. Yan M, Zhang Z, Cui S, et al. Preparation and evaluation of PEGylated phospholipid membrane coated layered double hydroxide nanoparticles. Asian J Pharm Sci. 2015;11(3):396-403.

30. Greish K, Thiagarajan G, Herd H, et al. Size and surface charge significantly influence the toxicity of silica and dendritic nanoparticles. Nanotoxicology. 2012;6(7):713-723.
International Journal of Nanomedicine

\section{Publish your work in this journal}

The International Journal of Nanomedicine is an international, peerreviewed journal focusing on the application of nanotechnology in diagnostics, therapeutics, and drug delivery systems throughout the biomedical field. This journal is indexed on PubMed Central, MedLine, CAS, SciSearch $®$, Current Contents $\AA /$ Clinical Medicine,

\section{Dovepress}

Journal Citation Reports/Science Edition, EMBase, Scopus and the Elsevier Bibliographic databases. The manuscript management system is completely online and includes a very quick and fair peer-review system, which is all easy to use. Visit http://www.dovepress.com/ testimonials.php to read real quotes from published authors. 